\title{
CHANGE OF TIME SCALE FOR MARKOV PROCESSES
}

BY

\author{
STEVEN OREY(1)
}

Let $\left\{X_{n}\right\}, n=0,1, \cdots$ be a Markov process with values in a measurable space $(S, B)$ and with a transition probability function $P(x, A)$. A measure $Q$ on $(S, B)$ is called invariant if

$$
Q(A)=\int_{S} P(x, A) Q(d x)
$$

for all $A \in B$.

Let $Q$ be an invariant measure. We assume the following conditions:

(i) $S$ is a locally compact topological space, $B$ is generated by the compact sets, and $Q$ is a regular measure.

(ii) $B$ is separable, $S \in \mathbb{B}, Q(S)>0$.

(iii) $Q$ is a sigma-finite invariant measure such that $P$ [entering $A$ at some time $\left.\mid X_{0}=x\right]=1$ for all $x \in S$ and all $A \in B$ with $Q(A)>0$, where $P$ is the underlying probability measure.

The conditional probability in (iii) is to be taken as determined by the transition probability function. The same remark applies to all similar situations appearing subsequently.

Processes satisfying (ii) and (iii) were introduced by Harris in [5] and studied further in $[7]\left({ }^{2}\right)$. Here we shall use the word recurrent for processes satisfying (i)-(iii). We continue to use $Q$ for some measure satisfying these conditions; in any case, according to [5] such a measure is unique up to constant multiple. fying

Let $\gamma(x)$ be a measurable function from $(S, B)$ into the positive reals satis-

$$
\int_{S} \gamma(x) Q(d x)<\infty
$$

Note that $\boldsymbol{\gamma}$ is required to be strictly positive.

Define the process $\{N(s)\}$ by

Received by the editors October $9,1959$.

(1) This research was supported by the United States Air Force through the Air Force Office of Scientific Research of the Air Research and Development Command, under Contract No. AF 49(638)-617. Reproduction in whole or in part is permitted for any purpose of the United States Government.

(2) In [7] these processes were referred to as recurrent Markov chains, but this may be misleading since chain often is reserved for processes with countable state space. 


$$
N(s)=\min _{k}\left\{k: \sum_{n=0}^{k} \gamma\left(X_{n}\right)>s\right\}, \quad s \geqq 0 .
$$

Then define $\bar{X}_{s}$ by

$$
\bar{X}_{\mathrm{s}}=X_{N(s)}, \quad s \geqq 0 .
$$

The $\left\{\bar{X}_{t}\right\}$ process, defined by $(0.2)$ is obtained from the $\left\{X_{n}\right\}$ process by requiring a wait of duration $\gamma(x)$ after arriving at $x$, for every $x \in S . \bar{X}_{t}$ is usually not a Markov process. There exists however a Markov process $\left\{\widetilde{X}_{t}\right\}$ such that

$$
\tilde{X}_{t}=\left(\bar{X}_{t}, Y_{t}\right)
$$

where $Y_{t}$ is the elapsed wait since the last transition.

We indicate the principal results of this paper. We consider a stationary Markov process $\left\{\tilde{X}_{t}^{*}\right\},-\infty<t<\infty$, where $\tilde{X}_{t}^{*}=\left(\bar{X}_{t}^{*}, Y_{t}^{*}\right)$, with the same transition probabilities as $\left\{\tilde{X}_{t}\right\}$ (defined by $(0.3)$ and more explicitly below). In a familiar way (see end of introduction) $\left\{\tilde{X}_{t}^{*}\right\}$ is associated with a group $\left\{U_{t}\right\}$ of unitary operators on a Hilbert space. In $\$ 1$ we show that the group of eigenvalues of $\left\{U_{t}\right\}$ is either trivial or it consists of all integral multiples of some $\lambda$. In the first case we say "condition $C$ holds." In $\$ 2$ we consider the case in which condition $C$ holds. We show that for every $z$ in the state space $P\left[\tilde{X}_{t}^{*} \in \cdot \mid \tilde{X}_{0}^{*}=z\right]$ converges, as $t \rightarrow \infty$, in a weak sense, made precise below. We use the approach of Doob [3], which is based on a mixing theorem for flows by von Neumann and Koopman. The case in which condition $\mathrm{C}$ does not hold is discussed in $\$ 3$. The appropriate convergence result in this case is shown to reduce to a result of [7]. Results in renewal theory are obtained in $\S 5$. Specifically it is shown that when 0 is the only eigenvalue of $\left\{U_{t}\right\}$ then the "renewal process" $\left\{Y_{t}\right\}$ converges in distribution. The usual renewal theory corresponds to the case in which the $\left\{X_{n}\right\}$ are a sequence of positive, independent, identically distributed random variables $\left({ }^{3}\right), \gamma(x)=x$, and $P\left[X_{0}<\alpha\right]=Q(\{x: 0<x<\alpha\})$. This situation is included in our discussion provided $E\left\{X_{0}\right\}<\infty$. Considering the "semi-Markov process" $\left\{\bar{X}_{t}\right\}$ we get immediately that, when condition $C$ holds, there exists a measure $\bar{Q}$ such that $P\left[\bar{X}_{t} \in A\right]$ converges as $t \rightarrow \infty$ to $\bar{Q}(A)$ for every $A \in B$. We show also, in $\S \S 4$ and 5 , that one can obtain similar results in case $\left\{\bar{X}_{t}\right\}$ is defined from $\left\{X_{n}\right\}$ by requiring a random wait of mean duration $\gamma(x)$ after reaching $x$; this extension was suggested by the work of Anselone [1].

From now on let $R^{+}$stand for the non-negative reals and let $D^{+}$be the Borel sets of $R^{+}$. Also, let $R, D$ stand for the real numbers and the Borel sets of real numbers. Let $\left(S^{*}, B^{*}\right)$ be the product space $(S, B) \times\left(R^{+}, D^{+}\right)$. Let $\tilde{S}$ consist of all pairs $(x, y)$ of $S^{*}$ such that $y<\gamma(x)$, and let $\tilde{B}$ consist of the subsets of $\tilde{S}$ belonging to $B^{*}$.

(3) In this case $\left\{Y_{t}\right\}$ is also a Markov process, and Doob exploited this fact in [4]. 
Let

$$
\tilde{X}_{\imath}=\left(X_{N(t)}, t-\sum_{n=0}^{N(t)-1} \gamma\left(X_{n}\right)\right)=\left(\bar{X}_{t}, Y_{t}\right) \text {, }
$$

where the last sum is to be interpreted as zero when $N(t)=0$. Then $\left\{\tilde{X}_{t}, t \geqq 0\right\}$ is a Markov process with transition probability function $P^{t}(z, B)$ defined for $z=(x, y) \in \tilde{S}, B \in \tilde{B}$ by the relations

$$
\begin{aligned}
& \left.P^{t}(z, B)=1(0) \text { for } 0 \leqq t<\gamma(x)-y \text { and }(x, y+t) \in B((x, y+t) \notin B)\right), \\
& P^{t}(z, B)=\sum_{n=1}^{\infty} \int_{\Gamma} P\left(x, x_{1}\right) P\left(x_{1}, x_{2}\right) \cdots P\left(x_{n-1}, x_{n}\right) d x_{1} d x_{2} \cdots d x_{n},
\end{aligned}
$$

where

$$
\Gamma=\left\{\left(x_{1}, \cdots, x_{n}\right):\left(x_{n}, t-\left(\gamma(x)-y+\sum_{i=1}^{n-1} \gamma\left(x_{i}\right)\right)\right) \in B\right\}
$$

The recurrence of $\left\{X_{n}\right\}$ insures that $P^{t}(z, \tilde{S})=1$ for $t \geqq 0, z \in \tilde{S}$.

It can be seen that $(Q \times m)$ is an invariant measure for $P^{t}$, where $m$ is Lebesgue measure. Clearly

$$
(Q \times m)(\tilde{S})=\int_{S} \gamma(x) Q(d x) .
$$

Let

$$
\tilde{Q}=[(Q \times m)(\tilde{S})]^{-1}(Q \times m) .
$$

Note that

$$
N(t)=\text { cardinal number of }\left\{s: Y_{\triangleleft}=0,0<s \leqq t\right\} .
$$

We shall write $P^{(n)}$ for the $n$-step transition probability function of the $\left\{X_{n}\right\}$ process.

There exists a Markov process $\left\{\tilde{X}_{t}^{*}\right\}$ having the following properties:

(a) The state space of $\left\{\tilde{X}_{t}^{*}\right\}$ is $(\tilde{S}, \tilde{Q})$ and the transition probabilities of $\left\{\tilde{X}_{t}^{*}\right\}$ are the same as those of $\left\{\tilde{X}_{t}\right\}$, i.e. $P^{t}(z, B)$ for $z \in \tilde{S}, B \in \tilde{B}$.

(b) $\left\{\tilde{X}_{t}^{*}\right\}$ is defined for $-\infty<t<\infty$.

(c) $\left\{\tilde{X}_{t}^{*}\right\}$ is stationary, so that $P\left[\tilde{X}_{t}^{*} \in B\right]=Q(B)$ for $B \in \tilde{B},-\infty<t<\infty$.

(d) The sample space $\Omega^{*}$ of $\left\{\tilde{X}_{t}^{*}\right\}$ consists exactly of all functions $\omega$ from $R$ into $S$ satisfying the following condition:

$$
\text { if } \omega(t)=(x, y) \text { and } 0 \leqq y+\epsilon<\gamma(x) \text { then } \omega(t+\epsilon)=(x, y+\epsilon) .
$$

(e) $\tilde{X}_{t}^{*}(\omega)=\omega(t)$.

The existence of a process satisfying (a)-(c) can be established by using 
Kolmogorov's extension theorem $\left({ }^{4}\right)$. Routine arguments can then be used to show that the process can be modified so as to satisfy (d) and (e) as well.

For each $z \in \tilde{S}$ there clearly exists a Markov process $\left\{\tilde{X}_{t}^{(z)}, t \geqq 0\right\}$ having the same paths and transition probabilities as $\left\{\tilde{X}_{t}^{*}, t \geqq 0\right\}$ and assigning measure 1 to the set of all paths $\omega$ such that $\omega(0)=z$. If $a^{+}$is the Borel field generated by $\left\{\tilde{X}_{t}^{*}, t \geqq 0\right\}$ there is a probability measure $P_{z}$ on $\left(\Omega^{*}, a^{+}\right)$corresponding to the $\left\{\tilde{X}_{t}^{(z)}\right\}$ process. If $A \in Q^{+}$we shall write $P\left[A \mid \tilde{X}_{0}^{*}=z\right]$ for $P_{z}(A)$; and a similar convention applies to conditioned expectations.

The shift by $t$ is defined as the transformation $T_{t}$ such that $\left(T_{t} \omega\right)(s)$ $=\omega(s+t)$ for all $\omega \in \Omega^{*}$ and $s \in R . T_{t}$ is a one-one measure preserving transformation on our probability space. Note that $\tilde{X}_{t}^{*}(\omega)=\tilde{X}_{0}^{*}\left(T_{t} \omega\right)$. It is easy to show that $T_{t} \omega$ is measurable as a function of $\omega$ and $t$.

We denote the two components of $\tilde{X}_{t}^{*}$ by $\bar{X}_{t}^{*}, Y_{t}^{*}$, i.e. $\tilde{X}_{t}^{*}=\left(\bar{X}_{t}^{*}, Y_{t}^{*}\right)$, and we define $N^{*}(t)$ by

$$
N^{*}(t)=\text { the cardinal number of }\left\{s: Y_{*}^{*}=0, \quad 0<s \leqq t\right\} .
$$

Although $\tilde{X}_{t}$ and $\tilde{X}_{t}^{*}$ are distinct processes their finite dimensional distributions conditioned by $\tilde{X}_{0}$ or $\tilde{X}_{0}^{*}$, respectively, obviously agree; the same applies to $\left\{\bar{X}_{t}\right\},\left\{Y_{t}\right\},\left\{N_{t}\right\}$ and $\left\{\bar{X}_{t}^{*}\right\},\left\{Y_{t}^{*}\right\},\left\{N_{t}^{*}\right\}$ (e.g. $E\left\{N(t) \mid \tilde{X}_{0}=z\right\}$ $\left.=E\left\{N^{*}(t) \mid \tilde{X}_{0}^{*}=z\right\}\right)$. Since we shall be interested only in such conditioned expectations and probabilities we shall henceforth omit the star. Actually from here through the end of $\$ 3$ all variables should be considered starred.

Let $a$ be the underlying Borel field on $\Omega^{*}$. If $\phi$ is a measure on $(\tilde{S}, \tilde{\beta})$ we define:

$$
P[\cdot \mid \phi]=\int_{\tilde{S}} P\left[\cdot \mid \tilde{X}_{0}=z\right] \phi(d z)
$$

and

$$
E\{\cdot \mid \phi\}=\int_{\tilde{S}} E\left\{\cdot \mid \tilde{X}_{0}=z\right\} \phi(d z)
$$

We write simply $P[\cdot], E\{\cdot\}$ for $P[\cdot \mid \widetilde{Q}], E\{\cdot \mid \widetilde{Q}\}$, respectively. In case $\phi$ is the probability measure on $(\tilde{S}, \tilde{B})$ concentrating its entire mass on the point $\boldsymbol{z}$ of $\tilde{S}$ we may write $P_{z}[\cdot], E_{z}\{\cdot\}$ for $P[\cdot \mid \phi], E\{\cdot \mid \phi\}$, respectively.

If $F$ is a random variable on $\left(\Omega^{*}, a\right), F_{t}$ is to be the random variable such that $F_{t}(\omega)=F\left(T_{t} \omega\right)$. Conversely, if the random variables $\left\{G_{t}\right\}$ are defined we may write $G$ for $G_{0}$. If $\phi$ is a measure on $(\tilde{S}, \tilde{\otimes})$ we define $\phi_{t}$ by

$$
\phi_{t}(A)=\int_{\tilde{s}} P\left[\tilde{X}_{t} \in A \mid \tilde{X}_{0}=z\right] \phi(d z)
$$

for $A \in B$. The basic fact to be exploited, of course, is that the $T_{t}$ are 1-1 and

( 4 Note that our topological assumptions allow us to apply Kolmogorov's theorem. 
measure preserving with respect to the measure $P[\cdot]$. On the complex $L_{2}$ space $\left(\Omega^{*}, a, P[\cdot]\right)$ the family of operators $\left\{U_{t}\right\}$ defined by

$$
U_{t} F=F_{t}, \quad t \in R
$$

is well known to constitute a group of unitary operators $\left({ }^{5}\right)$.

If $B \in \mathbb{B}, \tilde{B}$ is to be $\{(x, y): x \in B,(x, y) \in \widetilde{S}\}$.

1. The point spectrum of $\left\{U_{t}\right\}$. It is clear that the $\left\{\tilde{X}_{t}\right\}$ process is ergodic (because the $\left\{X_{n}\right\}$ process is recurrent), which implies that all eigenvalues of $\left\{U_{t}\right\}$ are simple (see $[6$, p. 30]).

Lemma 1. For all $z \in \tilde{S}$ the singular component of $P_{z}\left[\bar{X}_{t} \in \cdot\right]$ with respect to $Q$ approaches 0 as $t \rightarrow \infty$.

Proof. Let $z=(x, y) \in \tilde{S}, B \in B$ and $Q(B)=0$. Let $M$ be a positive integer and let $\epsilon>0$. For all big enough $t$ one has:

$$
\begin{aligned}
P_{z}\left[\bar{X}_{t} \in B\right] & =P_{z}\left(\bigcup_{n=0}^{\infty}\left[\bar{X}_{t} \in B, N(t)=n\right]\right) \\
& \leqq P_{z}\left(\bigcup_{n=M}^{\infty}\left[\bar{X}_{t} \in B, N(t)=n\right]\right)+\epsilon \\
& \leqq P\left(\bigcup_{n=M}^{\infty}\left[X_{n} \in B \mid X_{0}=x\right]\right)+\epsilon .
\end{aligned}
$$

As $M \rightarrow \infty$ the first term in the last expression must approach zero. For otherwise $P$ [entering $B$ infinitely often $\left.\mid X_{0}=x\right]>0$; but this is impossible, since $P$ [ever entering $B \mid X_{0}=u$ ] $=0$ outside some $Q$-null set of $u$ 's, say $C$, and by the recurrence $P$ [entering $\left.S-C \mid X_{0}=x\right]=1$. Since $\epsilon$ is arbitrary the lemma is established.

THEOREM 1. Either 0 is the only eigenvalue of $\left\{U_{t}\right\}$ or the set of eigenvalues consists of all integral multiples of a least positive eigenvalue $\lambda$. Every eigenvalue is simple.

Proof. The simplicity of the eigenvalues was noted above.

According to Doob $[3]\left({ }^{6}\right)$, for every eigenfunction $G_{t}(\omega)$ there exists a function $g$ on $\widetilde{S}$ such that $P\left[G_{t}=g\left(\tilde{X}_{t}\right), t \in R\right]=1$. So

$$
P\left[g\left(\tilde{X}_{\imath}\right)=e^{i \lambda t} g\left(\tilde{X}_{0}\right), t \in R\right]=1
$$

where $\lambda$ is the corresponding eigenvalue.

${ }^{(5)}$ For background in ergodic theory we refer to [6]; for material specifically involving Markov processes and ergodic theory see [3].

( $\left.{ }^{6}\right)$ Actually the theorem of [3] we cite asserts only that for each $t \in R P\left[G_{t}=g\left(\tilde{X}_{t}\right)\right]=1$. However for measurable flows one can obtain the identity (1.1); see [6, p. 27]. 
Remembering that the eigenvalues form an additive group (see [6, p. 27]) it is clear that the theorem will follow once we establish that there cannot exist arbitrarily small positive eigenvalues.

Let $\lambda$ be a positive eigenvalue and suppose (1.1) holds. The continuous component of $P^{(n)}(x, \cdot)$ with respect to $Q$ has a density, which we denote by $p^{n}(x, \cdot)$. Let $C^{\prime} \in \beta$ be such that $Q\left(C^{\prime}\right)>0$ and for some positive integer, say $n, p^{n}(x, u)>0$ for all $x, u \in C^{\prime}$; it was shown in [7] that the existence of such a $C^{\prime}$ follows from the fact that $\left\{X_{n}\right\}$ is recurrent. An easy argument shows the existence of a subset $C$ of $C^{\prime}$ of positive $Q$-measure and of a number $M$ such that

$$
P_{z}\left[\tilde{X}_{t} \in A \text { for some positive } t \text { less than } M\right]>0
$$

whenever $z \in \tilde{C}, A \subseteq \tilde{C}, \widetilde{Q}(A)>0$. Here $M$ does not depend on $\lambda$. We may and shall suppose $|g(z)|$ to be constant on $\tilde{S}$. For $z_{1}, z_{2} \in \tilde{S}$ let $h\left(z_{1}, z_{2}\right)$ be the least non-negative number $h$ such that

$$
g\left(z_{2}\right)=\exp \left\{i \lambda h\left(z_{1}, z_{2}\right)\right\} g\left(z_{1}\right) .
$$

We can therefore write

$$
g\left(z_{2}\right)=\exp \left\{i \lambda\left[h\left(z_{1}, z_{2}\right)+h\left(z_{2}, z_{1}\right)\right]\right\} g\left(z_{2}\right) .
$$

A simple argument using (1.1) and (1.2) shows that for $\widetilde{Q}$-almost all $z_{1} \in \tilde{C}$ $\tilde{Q}\left\{z_{2} \in \tilde{C}: h\left(z_{1}, z_{2}\right) \geqq M\right\}=0$. So there exist $z_{1}$ and $z_{2}$ such that $h\left(z_{2}, z_{1}\right)<M$ and $0<h\left(z_{1}, z_{2}\right)<M$. For such $z_{1}$ and $z_{2}$ (1.4) shows that there exists a positive integer $k$ such that

$$
\lambda\left(h\left(z_{1}, z_{2}\right)+h\left(z_{2}, z_{1}\right)\right)=2 \pi k \geqq 2 \pi
$$

so that

$$
\lambda \geqq \pi / M \text {. }
$$

2. Zero the only eigenvalue. In this section we study the case in which the following condition is satisfied:

The point spectrum of $\left\{U_{t}\right\}$ reduces to $\{0\}$.

The mixing theorem of von Neumann and Koopman states that (C) is equivalent to the following condition:

There is a subset $W$ of $R^{+}$with relative measure zero such that for any two complex valued random variables $F$ and $G$ satisfying $E\left\{|F|^{2}\right\}<\infty$, $E\left\{|G|^{2}\right\}<\infty, E\left\{F_{t} G\right\} \rightarrow E\{F\} E\{G\}$ as $t \rightarrow \infty$ avoiding $W$.

From here up to the end of this section $W$ is to be some fixed set satisfying the conditions of the mixing theorem. We write " $\phi \ll \psi$ " for " $\phi$ is absolutely continuous with respect to $\psi$."

LEMMA $2.1\left(^{7}\right)$. Let $(\mathrm{C})$ hold. Let $F$ be a complex valued random variable on

(7) This lemma is due to Doob; see [3] 
$(\Omega, a)$ satisfying the following condition: there exist numbers $M$ and $t_{0}$ such that $E\left\{\left|F_{t}\right| \mid z\right\} \leqq M$ for all $t>t_{0}$ and all $z \in \tilde{S}$. Let $\phi$ be a probability measure on $(\tilde{S}, \tilde{Q})$ satisfying $\phi \ll \tilde{Q}$. Then $E\left\{F_{t} \mid \phi\right\} \rightarrow E\{F\}$ as $t \rightarrow \infty$ avoiding $W$.

Proof. Let $p(\cdot)$ be the density of $\phi$ with respect to $\tilde{Q}$. Then

$$
\begin{aligned}
E\left\{F_{t} \mid \phi\right\} & =\int_{S} E\left\{F_{t} \mid \tilde{X}_{0}=z\right\} p(z) \widetilde{Q}(d z) \\
& =E\left\{E\left\{F_{t} \mid \tilde{X}_{0}\right\} p\left(\tilde{X}_{0}\right)\right\}=E\left\{F_{t} p\left(\tilde{X}_{0}\right)\right\} .
\end{aligned}
$$

If $E\left\{\left(p\left(\tilde{X}_{0}\right)\right)^{2}\right\}<\infty$ the mixing theorem applies; otherwise first approximate $p\left(\tilde{X}_{0}\right)$ by a square integrable function.

For $\delta \geqq 0$ define

$$
\begin{aligned}
& U(\delta)=\{(x, y) \in \tilde{S}: \gamma(x)-\delta \leqq y<\gamma(x)\}, \\
& L(\delta)=\{(x, y) \in \tilde{S}: 0 \leqq y<\delta\} .
\end{aligned}
$$

LeMma 2.2. Let $(\mathrm{C})$ hold. Let $\phi$ be a probability measure on $(\tilde{S}, \tilde{\mathbb{B}})$ such that $P\left[\bar{X}_{0} \in \cdot \mid \phi\right] \ll Q$. Then for $\beta>0, \quad P\left[\tilde{X}_{\iota} \in U(\beta) \mid \phi\right] \rightarrow \Phi(U(\beta))$ and $P\left[\tilde{X}_{t} \in L(\beta) \mid \phi\right] \rightarrow \widetilde{Q}(L(\beta))$ as $t \rightarrow \infty$ avoiding $W$.

Proof. It will suffice to prove the lemma under the additional assumption that $\phi(U(\delta))=0$ for some $\delta>0$, since $\phi$ can be approximated in variation by measures satisfying this condition as well as the other conditions on $\phi$. So assume $\phi(U(\bar{\delta}))=0,0<\bar{\delta}<\beta$. Let $0<\delta<\bar{\delta}$ and let $\left.{ }^{8}\right)$

$$
\phi_{(\delta)}=\frac{1}{\delta} \int_{0}^{\delta} \phi_{\eta} d \eta .
$$

One can see that $\phi_{(\delta)} \ll \tilde{Q}$. For $0<\eta<\delta$ one has

$$
\begin{aligned}
P\left[\tilde{X}_{t} \in U(\beta-\delta) \mid \phi_{\eta}\right] & =P\left[\tilde{X}_{t+\eta} \in U(\beta-\delta) \mid \phi\right] \\
& \leqq P\left[\tilde{X}_{t} \in U(\beta) \mid \phi\right] \leqq P\left[\tilde{X}_{t+\eta} \in U(\beta) \cup L(\delta) \mid \phi\right] \\
& =P\left[\tilde{X}_{t} \in U(\beta) \cup L(\delta) \mid \dot{\phi}_{\eta}\right] .
\end{aligned}
$$

Therefore

$$
\begin{aligned}
P\left[\tilde{X}_{t} \in U(\beta-\delta) \mid \phi_{(\delta)}\right] & \leqq P\left[\tilde{X}_{t} \in U(\beta) \mid \phi\right] \\
& \leqq P\left[\tilde{X}_{t} \in U(\beta) \cup L(\delta) \mid \phi_{(\delta)}\right]
\end{aligned}
$$

Let $t \rightarrow \infty$ avoiding $W$ and note that Lemma 2.1 applies with $\phi_{(\delta)}$ in place of $\phi$; then let $\delta \rightarrow 0$ to obtain the desired conclusion for $U(\beta)$. The $L(\beta)$ part is proved similarly.

Using the discrete topology for $S$ and the ordinary topology for $R^{+}$, we may introduce the product topology on $S \times R^{+}$; this induces a topology on $\widetilde{S}$.

(8) Remember that $\phi_{q}(A)=\int \check{s} P\left[\tilde{X}_{4} \in A \mid \tilde{X}_{0}=z\right] \phi(d z)$. 
This topology will be used from now on. Note that a function $f((x, s))$ is continuous if and only if $f((x, s))$ is continuous in $s$ for every $x$.

LEMMA 2.3. Let (C) hold. Let $f$ be a measurable, complex valued function on $\tilde{S}$ which is continuous and bounded in absolute value. Let $\phi$ be a probability measure on $(\tilde{S}, \tilde{B})$ such that $P\left[\bar{X}_{0} \in \cdot \mid \phi\right] \ll Q$. Let $F_{t}=f\left(\tilde{X}_{t}\right)$. Then $\left({ }^{9}\right) E\left\{F_{t} \mid \phi\right\}$ $\rightarrow E\{F\}$ as $t \rightarrow \infty$ avoiding $W$.

Proof. Suppose first that $f$ is real valued. Let $\beta>0$ and let $g$ be a real valued function on $\tilde{S}$ satisfying the same conditions as $f$, vanishing on $L(\beta / 2) \cup U(\beta / 2)$ and agreeing with $f$ on $S-(L(\beta) \cup U(\beta))$. Let $G_{t}=g\left(\tilde{X}_{t}\right)$. Since $\beta$ is arbitrary we can use Lemma 2.2 to infer the conclusion of the present lemma for $F_{t}$ once we have proved it for $G_{t}$. Again it can and will be assumed that $\phi(U(\bar{\delta}))=0$, where $\bar{\delta}>0$. Let $0<\delta<\min (\bar{\delta}, \beta / 2)$ and define

$$
\begin{array}{rlrl}
g^{* \delta}((x, y)) & =g^{* * \delta}((x, y))=0 & \text { for }(x, y) \in L(\delta), \\
g^{* \delta}((x, y))=\inf _{0 \leqq \eta \leqq \delta} g((x, y-\eta)) & \text { for }(x, y) \in \tilde{S}-L(\delta), \\
g^{* * \delta}((x, y))=\sup _{0 \leqq \eta \leqq \delta} g((x, y-\eta)) & \text { for }(x, y) \in \tilde{S}-L(\delta), \\
G_{t}^{* \delta}=g^{* \delta}\left(\tilde{X}_{t}\right), \quad G_{t}^{* * \delta}=g^{* * \delta}\left(\tilde{X}_{t}\right) . &
\end{array}
$$

Then for $0 \leqq \eta \leqq \delta$ one has

$$
\begin{aligned}
E\left\{G_{t}^{* \delta} \mid \phi_{\eta}\right\} & =E\left\{G_{t+\eta}^{* \delta} \mid \phi\right\} \leqq E\left\{G_{t} \mid \phi\right\} \\
& \leqq E\left\{G_{t+\eta}^{* * \delta} \mid \phi\right\}=E\left\{G_{t}^{* * \delta} \mid \phi_{\eta}\right\}
\end{aligned}
$$

Therefore

$$
\left.E\left\{G_{t}^{* \delta} \mid \phi_{(\delta)}\right)\right\} \leqq E\left\{G_{t} \mid \phi\right\} \leqq E\left\{G_{t}^{* * \delta} \mid \phi_{(\delta)}\right\} .
$$

As $T \rightarrow \infty$ avoiding $W$, Lemma 2.1 , with $\phi_{(\delta)}$ in place of $\phi$, shows that the first and last members of (2.1) approach $E\left\{G^{* 8}\right\}$ and $E\left\{G^{* * \delta}\right\}$ respectively. The continuity and boundedness of $g$ allow application of the bounded convergence theorem to conclude that as $\delta \rightarrow 0$ the common limit of $E\left\{G^{* \delta}\right\}$ and $E\left\{G^{* * \delta}\right\}$ is $E\{G\}$. The theorem now follows under the assumption we made that $f$ is real valued. If $f$ is not real valued the desired result follows by considering the real and imaginary part separately.

Lemma 2.4. Let (C) hold. Let $f, F_{t}$ be as in the preceding lemma. Let $\phi$ be a probability measure on $(\tilde{S}, \tilde{B})$. Then $E\left\{F_{t} \mid \phi\right\} \rightarrow E\{F\}$ as $t \rightarrow \infty$ avoiding $W$.

Proof. One has for $t \geqq u \geqq 0$

(') Remember that $F=F_{0}=f\left(\tilde{X}_{0}\right)$. 


$$
\begin{aligned}
E\left\{F_{t} \mid \phi\right\} & =\int_{\tilde{S}} E\left\{F_{t} \mid \tilde{X}_{u}=z\right\} P\left[\tilde{X}_{u} \in d z \mid \phi\right] \\
& =\int_{\tilde{S}} E\left\{F_{t} \mid \tilde{X}_{u}=z\right\} \phi_{u}(d z) .
\end{aligned}
$$

If $\psi$ is a probability measure on $(\tilde{S}, \tilde{B})$ such that $P\left[\bar{X}_{0} \in \cdot \mid \psi\right] \ll Q$, then we claim,

$$
\int_{\tilde{S}} E\left\{F_{t} \mid \tilde{X}_{u}=z\right\} \psi(d z) \rightarrow E\{F\} \quad \text { as } t \rightarrow \infty
$$

avoiding $W$. In case $u=0$ this is the assertion of the previous lemma; for $u>0$ one proves the assertion in the same way as for $u=0$. So the lemma would follow from (2.2) if for some $u P\left[\bar{X}_{0} \in \cdot \mid \phi_{u}\right] \ll Q$; but for large $u$ the last relation is almost true, in a sense made precise by Lemma 1 . The lemma now follows.

THEOREM $2\left({ }^{10}\right)$. Let $(\mathrm{C})$ hold. Let $\mathrm{g}$ be a complex valued measurable function on $(\tilde{S}, \tilde{\otimes})$ which is continuous and bounded in absolute value. Let $\phi$ be a proba. bility measure on $(\tilde{S}, \tilde{B})$. Let $G_{t}=g\left(\tilde{X}_{t}\right)$. Then $E\left\{G_{t} \mid \phi\right\} \rightarrow E\{G\}$ as $t \rightarrow \infty$.

Proof. Let $\left\{t_{n}\right\}$ be a sequence of non-negative reals tending to infinity with $n$. For $n=0,1, \cdots$, chose $u_{n} \in R^{+}$such that $t_{n}-u_{n} \notin W, u_{n} \notin W, u_{n}<t_{n}$, and $t_{n}-u_{n} \rightarrow \infty, u_{n} \rightarrow \infty$ as $n \rightarrow \infty$. Then

$$
\begin{aligned}
E\left\{G_{t_{n}} \mid \phi\right\} & =\int_{\tilde{S}} E\left\{G_{t_{n}} \mid \tilde{X}_{u_{n}}=z\right\} \phi_{u_{n}}(d z) \\
& =\int_{\tilde{S}} E\left\{G_{t_{n}-u_{n}} \mid \tilde{X}_{0}=z\right\} \phi_{u_{n}}(d z) .
\end{aligned}
$$

According to Lemma 2.4 one has, for every $z \in \tilde{S}$,

$$
E\left\{G_{t_{n-u_{n}}} \mid \tilde{X}_{0}=z\right\} \rightarrow E\{G\} \quad \text { as } n \rightarrow \infty .
$$

Applying Egoroff's theorem to (2.4) allows us to break the last integral in (2.3) up into two integrals, one extending over a set $B$, the other over $\widetilde{S}-B$, where $Q(B)$ can be made less than any arbitrary $\epsilon>0$, and the integrand converges uniformly on $\tilde{S}-B$. The theorem would now follow if we knew that $\phi_{u_{n}}(B) \rightarrow \widetilde{Q}(B)$ with $n$. Note that we may choose $B$ to be open. A simple approximation proves that characteristic functions of open sets satisfy the conclusion of Lemma 2.4. From this the theorem follows.

$\left.{ }^{10}\right)$ Note that a simple approximation argument shows that the theorem remains true if the requirement that $g$ is continuous is replaced by the condition that for every $x \in S$ the set of discontinuities of $g(x, s)$ has Lebesgue measure zero. 
3. More than one eigenvalue. We now consider the case in which (C) does not hold. By Theorem 1 there exists a least positive eigenvalue $\lambda$. Then there exists a complex valued measurable function $g$ on $(\tilde{S}, \tilde{\mathbb{B}})$ such that $P\left[g\left(\tilde{X}_{t}\right)=e^{i \lambda t} g\left(\tilde{X}_{0}\right), t \in R\right]=1$. Let

$$
C=\left\{z \in \tilde{S}: P\left[g\left(\tilde{X}_{t}\right)=e^{i \lambda t} g\left(\tilde{X}_{0}\right), t \in R \mid \tilde{X}_{0}=z\right]=1\right\} .
$$

Let $h=2 \pi / \lambda$. Let $z_{0} \in C$, and define

$$
C_{s}=\left\{z \in C: g(z)=e^{i \lambda_{s}} g\left(z_{0}\right)\right\} \text {. }
$$

It is clear that $C$ differs from $U C_{s}$ by a $\tilde{Q}$-null set, the union extending over $0 \leqq s<h$; also clearly $\widetilde{Q}(C)=1$. Furthermore $P\left[\tilde{X}_{t} \in C\right.$ for all sufficiently large $\left.t \mid \tilde{X}_{0}=z\right]=1$ for all $z \in \tilde{S}$. Let $0 \leqq s<h$. It follows from the fact that $P\left[g\left(\tilde{X}_{t}\right)=e^{i \lambda t} g\left(\tilde{X}_{0}\right), t \in R\right]=1$ that with probability one $\tilde{X}_{t}(\omega)$ enters $C_{s}$ only a denumerable number of times, say at $\tau_{1}, \tau_{2}, \ldots$. In the terminology of [7] $\left\{\tilde{X}_{\tau_{n}}\right\}, n=0,1, \cdots$ is "the process on $C_{8}$ " and this is a recurrent acyclic Markov process with finite invariant measure. Since the parameter is now discrete the convergence result of [7] applies, and the following theorem is easily established.

TheOREM 3. Let $\phi$ be any probability distribution on $(\tilde{S}, \tilde{B})$. Then $P\left[\tilde{X}_{n h+\delta} \in \cdot \phi\right]$ converges in variation as $n \rightarrow \infty$ uniformly for $0 \leqq \delta<h$.

It appears that in the present case we have a result in some respect stronger than that for the acyclic case, since here we have convergence in variation.

4. Random waits. We shall extend the results of the last sections. The generalization to be considered suggested itself to us after we read the abstract of Anselone [1].

The process $\left\{\bar{X}_{t}\right\}$ was originally obtained from the Markov process $\left\{X_{n}\right\}$ by requiring a wait of duration $\gamma(x)$ after reaching $x \in \tilde{S}$. We now consider the case where the waiting time after reaching $x$ is itself a random variable.

Let

$$
\begin{aligned}
\bar{X}_{\lambda W_{0}}=X_{0}, & 0 \leqq \lambda<1, \\
\bar{X}_{W_{0}+\cdots+W_{n-1}+\lambda W_{n}}=X_{n}, & 0 \leqq \lambda<1, \quad n=1,2, \cdots,
\end{aligned}
$$

where $W_{0}, W_{1}, \cdots$, is a sequence of positive random variables such that

$$
P\left[W_{n} \leqq \alpha \mid X_{n}, X_{n-1}, \cdots X_{0}, W_{n-1}, \cdots, W_{0}\right]=P\left[W_{n} \leqq \alpha \mid X_{n}\right]
$$

and

$$
P\left[W_{n} \leqq \alpha \mid X_{n}=x\right]=P\left[W_{m} \leqq \alpha \mid X_{m}=x\right\rfloor
$$

for any $\alpha \geqq 0, x \in S$, and any non-negative integers $n$ and $m$. We write

$$
F_{x}(\alpha) \text { for } P\left[W_{n} \leqq \alpha \mid X_{n}=x\right]
$$

and 


$$
\gamma(x) \text { for } \int_{0}^{\infty} s F_{x}(d s)
$$

We assume that $\gamma(x)<\infty$ for every $x \in S$ and

$$
\int_{S} \gamma(x) Q(d x)<\infty
$$

Now we introduce $\left\{\tilde{X}_{t}\right\}$ as follows. Let $\left(S^{*}, B^{*}\right)$ be the product space $(S, B) \times\left(R^{+}, D^{+}\right) \times\left(R^{+}, D^{+}\right)$.

Let $\tilde{S}=\left\{(x, y, z) \in S^{*}: y=0\right.$ and $z=0$, or $\left.0<z<y\right\}$ and let $\tilde{B}$ consist of all $B \in B^{*}$ such that $B \subseteq \tilde{S}$. The $\left\{\tilde{X}_{t}\right\}$ process has $(\tilde{S}, \tilde{B})$ as state space; and it behaves as follows: after arriving at a point $(x, 0,0)$ it spends the next $y$ time units moving up the "line" $\{(x, y, z): 0<z<y\}$ at a constant rate $\left.{ }^{11}\right)$; here $y$ is selected according to the distribution $F_{x}(\cdot)$. Then the process jumps back down to $\left(x^{\prime}, 0,0\right)$, where the probability that $x^{\prime} \in E$ is $P^{1}(x, E)$ for any $E \in ß$. The $\left\{\tilde{X}_{t}\right\}$ process is again Markov, and it has an invariant measure $\tilde{Q}$. Note that if $E \in \tilde{B}$ has the form $E=\{(x, y, z) \in \tilde{S}: x \in A\}$, for $A \in S$, then still

$$
\widetilde{Q}(E)=c \int_{A} \gamma(x) Q(d x)
$$

where $c$ is a fixed constant.

In the present case we introduce on $\tilde{S}$ a topology such that a function $f((x, y, z))$ on $\tilde{S}$ is continuous if and only if it is continuous as a function of $z$ for $0 \leqq z<y$ (using the standard topology for the reals) for every $x \in S$ and $y>0$.

One easily verifies the following result:

THEOREM 4. Theorems 1-3 remain true for the more general $\left\{X_{t}\right\}$ process introduced in this section.

5. Applications. In this section we consider some applications of the theorems of the previous sections.

RENEWAL THEORY. In case $\left\{X_{n}\right\}$ is stationary the ergodic theorem asserts that

$$
P\left[\frac{1}{n} \sum_{k=0}^{n} \gamma\left(X_{k}\right) \rightarrow E\left\{\gamma\left(X_{0}\right)\right\} \text { as } n \rightarrow \infty\right]=1
$$

from which one easily obtains

$$
P\left[\frac{N(t)}{t} \rightarrow \frac{1}{E\left\{\gamma\left(X_{0}\right)\right\}} \text { as } t \rightarrow \infty\right]=1 .
$$

(11) That is to say, $z$ grows linearly up to $y$ and then falls lack to zero. 
In case the limit may be taken outside the integral this leads to

$$
\frac{E\{N(t)\}}{t} \rightarrow \frac{1}{E\left\{\gamma\left(X_{0}\right)\right\}}
$$

as $t \rightarrow \infty$.

The step from (5.1) to (5.2) will always be justified if

$$
\text { inf } \gamma>0 \text {, }
$$

for then the bounded convergence theorem applies.

Our discussion in this section so far has made no use of the Markov property of $\left\{X_{n}\right\}$; it applies to any stationary process.

We continue to take $\left\{X_{n}\right\}$ to be a recurrent Markov process, not necessarily stationary. $\widetilde{Q}$ is the stationary initial probability distribution for $\left\{\tilde{X}_{t}\right\}$.

Our results will be stated under the assumption (C); in view of Theorems 1 and 3 one easily obtains modified results when (C) fails to hold, that is, in the "cyclic case."

Note that Theorem 2 tells us that when (C) holds $\left\{Y_{t}\right\}$ (the "renewal process") converges in distribution. Let $f(\Delta,(x, s))=E\left\{N(\Delta) \mid \tilde{X}_{0}=(x, s)\right\}$. For every $x \in S, f(\Delta,(x, s))$ is a monotone nondecreasing function on $0 \leqq s$ $<\gamma(x)$, so that it satisfies the condition of footnote 4 . Let $F_{t}(\Delta)=f\left(\Delta, \tilde{X}_{t}\right)$. If (C) holds and for some (and therefore every) $\Delta \in R^{+}$there exists a constant $M$ such that

$$
E\left\{N(\Delta) \mid \tilde{X}_{0}=z\right\}<M \Delta \quad \text { for all } z \in \tilde{S}
$$

holds, then for every probability measure $\phi$ on $(\tilde{S}, \tilde{\mathbb{B}})$ and every $\Delta \in R^{+}$

$$
\begin{aligned}
E\left\{F_{t}(\Delta) \mid \phi\right\} & =E\{N(t+\Delta)-N(t) \mid \phi\} \\
& \rightarrow E\{F(\Delta) \mid \tilde{Q}\}=E\{N(\Delta)\}=k \Delta
\end{aligned}
$$

where $k$ is a certain positive constant. The convergence assertion follows from Theorem 2 and footnote 10, and the last equality from the fact that the stationarity makes $E\{N(\Delta)\}$ a linear function of $\Delta$.

We have not assumed that the process $\left\{X_{n}\right\}$ has a stationary probability measure (but it will shortly appear that when $\widetilde{Q}$ is finite, which we have assumed, (5.4) can hold only when $Q$ is finite) $\left({ }^{12}\right)$; but consider now the case in which

$$
Q(S)=1 .
$$

We shall show that it follows from (5.6) that

$$
k=\left[\int_{s} \gamma(x) Q(d x)\right]^{-1}
$$

in formula (5.5).

(12) We call a measure finite (infinite) if it assigns finite (infinite) measure to the whole space. 
Again let $B(\boldsymbol{\epsilon})=\{x \in S: \gamma(x) \geqq \epsilon\}$. Let $B_{0}(\boldsymbol{\epsilon})=B(\boldsymbol{\epsilon}), B_{1}(\boldsymbol{\epsilon})=S-B(\boldsymbol{\epsilon})$, and let for $i=0,1$

$N^{(i)}(\epsilon, \delta)=$ the cardinal number of $\left\{s: 0<s \leqq \delta, Y_{s}=0, \bar{X}_{s} \in B_{i}(\epsilon)\right\}$

$$
N(\delta)=N^{(0)}(\epsilon, \delta)+N^{(1)}(\epsilon, \delta) \quad \text { for } \epsilon \geqq 0, \delta \geqq 0 .
$$

Let $\epsilon>0$, and choose $\delta$ so that $E\left\{N^{(1)}(\delta, 1)\right\}<\epsilon$; that this can be done is a consequence of (5.4). Then

$$
E\left\{N^{(1)}(\delta, \delta)\right\}<\epsilon \delta
$$

On the other hand

$$
E\left\{N^{0}(\delta, \delta)\right\}=P\left[\tilde{X}_{\delta} \in L(\delta) \cap \widetilde{B}_{0}(\delta)\right]
$$

$$
=\widetilde{Q}\left(L(\delta) \cap \tilde{B}_{0}(\delta)\right)=\delta \cdot Q\left(B_{0}(\delta)\right)\left[\int_{S} \gamma(x) Q(d x)\right]^{-1}
$$

the last equality being a consequence of (0.4) and (0.5). Since $\epsilon$ is arbitrary and $B_{0}(\delta) \rightarrow S$ as $\delta \rightarrow 0$ (5.7) follows from (5.8), (5.9) and (5.10).

In case $Q$ is infinite arguments similar to the above show that $E\left\{F_{t}(\Delta) \mid \phi\right\}$ $\rightarrow \infty$ as $t \rightarrow \infty$ when (C) holds.

To summarize: Conditions (C), (5.4), and (5.6) imply (5.5) with $k$ being given by (5.7). If (C) holds and $Q$ is infinite the limit in (5.5) is infinite.

Note that (5.3) implies (5.4).

In the case in which $\left\{X_{n}\right\}$ is a sequence of identically distributed independent random variables (C) automatically holds unless the variables $\gamma\left(X_{n}\right)$ are lattice variables, (5.4) is always true, and so is (5.6), since for $Q$ one has the distribution function of the $X_{n}$. In this case our conclusion that (5.5) and (5.7) hold is Blackwell's renewal theorem $\left({ }^{13}\right)$; however we have assumed (0.1) throughout, so that our results do not include that part of Blackwell's theorem asserting that when (0.1) is violated (5.5) holds with $k=0$.

Another consequence of Theorem 2 is that when $(C)$ holds, we have for every probability measure $\phi$ on $(\widetilde{S}, \widetilde{B})$

$$
E\left\{e^{i[N(t+\Delta)-N(t)] u} \mid \phi\right\} \rightarrow E\left\{e^{i N(\Delta) u} \mid \tilde{Q}\right\}
$$

as $t \rightarrow \infty$

so that the distribution of $N(t+\Delta)-N(t)$ converges weakly as $t \rightarrow \infty$.

Note that for (5.11) no condition other than (C) is necessary. It is easy to give an example of a recurrent Markov chain $\left\{X_{n}\right\}$ and a $\gamma$ such that $\left\{X_{n}\right\}$ is stationary with invariant probability measure $Q$ (the state space may even be denumerable), (0.1) holds, (C) holds, and $E\{N(\Delta)\}=\infty$; nevertheless (5.1) and (5.11) will hold.

Change of SCAlE. Starting with the Markov process $\left\{X_{n}\right\}$ we obtained $\left\{\bar{X}_{t}\right\}$ by a change of scale, that is, in the present case, by requiring a certain (13) See [2]. 
wait after arriving at a state where the waiting time will depend on the state and may be, according to $\$ 4$, a random variable. In view of the topology used on $\tilde{S}$ the convergence results for $\left\{\bar{X}_{t}\right\}$ are stronger than those for $\left\{Y_{t}\right\}$.

In case $(C)$ holds we have for $B \in B$

$$
P\left[\bar{X}_{t} \in B\right] \rightarrow \bar{Q}(B)
$$

as $t \rightarrow \infty$.

Here $\bar{Q}$ may of course be infinite. E.g. let $\left\{X_{n}\right\}$ be the symmetric random walk on the integers and obtain $\bar{X}_{t}$ by introducing a (random) waiting time of (mean) duration $\gamma(k)$ after arriving at $k$. Assume

$$
\sum_{k=-\infty}^{\infty} \gamma(k)=c<\infty .
$$

Then, if (C) holds, $P\left[\bar{X}_{t}=k\right] \rightarrow \gamma(k) / c$ as $t \rightarrow \infty$.

\section{BIBLIOGRAPHY}

1. P. M. Anselone, Ergodic theorems for discrete semi-Markov processes, Duke Math. J. vol. 27 (1960) pp. 33-40.

2. D. Blackwell, $A$ renewal theorem, Duke Math. J. vol. 15 (1948) pp. 145-151.

3. J. L. Doob, Asymptotic properties of Markoff transition probabilities, Trans. Amer. Math. Soc. vol. 63 (1948) pp. 293-321.

4. - Renewal theory from the point of view of the theory of probability, Trans. Amer. Math. Soc. vol. 63 (1948) pp. 422-438.

5. T. E. Harris, The existence of stationary measures for certain Markov processes, Third Berkeley Symposium on Mathematical Statistics and Probability, Vol. III, Berkeley, 1956, pp. 113-124.

6. E. Hopf, Ergodentheorie, Berlin, Julius Springer, 1937.

7. S. Orey, Recurrent Markov chains, Pacific J. Math. vol. 9 (1959) pp. 805-827.

UNIVERSITY OF MiNNESOTA, Minneapolis, Minnesota 